\title{
Promover la lectura de obras teatrales a través del blog docente Teatro en red
}

Número 5.

Enero de 2016

\author{
Alfredo Blanco Martínez
}

Universidad de La Coruña
Pág. 44 a la 51

\section{Keywords}

theatre, blog, ICT, qualitative research

\begin{abstract}
:
Nowadays, schools have a project for young people to acquire a taste and a habit of reading. However, it is also necessary that the educational community is configured as an important piece of the network of reading. For this reason, it is essential to show young people a variety of literature texts to learn and appreciate all literary genres. Probably, the play is the least consumed by teenagers, because they associate that kind of text to the theatrical production, and also, they omit the pleasure of reading it. In this sense, it is important that teachers, professionals and families promote reading plays. In this paper, we examine and describe the project Teatro en red from the perspective of its founders. The teacher blog has the aim to disseminate contemporary theatre and, at the

Specifically, we will focus on promoting reading and we will make a special emphasis on educational and cultural strategies pursued by the blog. In this case, we have used a qualitative methodology to make, on the one hand, a holistic understanding of the subject matter and, secondly, to respond to objectives of the study. With respect to the techniques of data collection, we have used the interview to reveal the voices of two teachers and, also, we have utilised the document analysis to know in depth the blog. For analysis of the data collected, we have started a systematic approach and, also, we have used a process of encoding to set the dimensions and categories that illustrate the work of teachers. In conclusion, we will discover that the blog is a social tool accessible to tell stories and, also, to form a reader taste in theatre between young people.
\end{abstract} same time, it expects to bring young people to the dramatic world from different perspectives. 



\section{Justificación}

Uno de los pilares de la arquitectura educativa está en estrecha relación con el ámbito lector y su respectiva promoción entre los jóvenes estudiantes, así como entre los profesionales de la educación. Este propósito cobra fuerza en el marco legislativo y curricular de España y, al mismo tiempo, cobra vida a través de los proyectos lectores de centro de cada Comunidad Autónoma.

No obstante, a pesar de que la iniciativa tiene repercusiones positivas sobre el alumnado, no siempre se logra que adquieran un suficiente bagaje cultural y lector. En consecuencia, el estudiante aterriza sobre un terreno árido y, por tanto, no es capaz de descubrir por sí solo la totalidad de los géneros literarios, así como distintas vías, tanto tradicionales como electrónicas, para la lectura de los mismos.

Actualmente, la novela ocupa el primer puesto en el ranking de lectura y tiene un papel social con repercusiones educativas entre el público lector. Sin embargo, otros géneros, como la poesía y el teatro, son menos demandados y, aparentemente, sin motivo alguno. En lo que respecta a nuestro tema de estudio y en línea con Pérez Fernández (2011), es necesario concienciar a la masa social del carácter atractivo y motivador del texto teatral. Se trata de un género que posibilita vivir dos experiencias: la visual a través del espectáculo y de la representación; y el viaje interior a través del texto.

De acuerdo con Díez Mediavilla y García Velasco (2015), hay que proponer soluciones para que los jóvenes desarrollen una competencia lectora en diferentes ámbitos y lugares, como puede ser en los entornos virtuales. De esta manera, se puede conseguir que, desde cualquier espacio, el alumno/a adquiera los niveles derivados de la educación lectora: nivel literal, nivel representativo, nivel inferencial, nivel crítico, nivel emocional, nivel creador y nivel metacognitivo (Jiménez Pérez, 2015).

Una posibilidad de activar la iniciación lectora en teatro es a través de los recursos TIC, dado su impacto en la sociedad y su inminente carácter didáctico (Cabero, 2010). En los últimos años, han emergido distintos recursos web y redes sociales que permiten una mayor difusión y lectura de teatro.

En este caso, nos centramos en el uso del blog como espacio para la promoción lectora. Asumiendo las afirmaciones de Amarós Poveda (2009), Hernández Serrano y González Sánchez (2014), el blog se concibe como un recurso altamente pedagógico con oportunidades formativas tanto para el docente como para el alumnado. Se trata de un lienzo en blanco capaz de almacenar y compartir información, así como de establecer vínculos virtuales con otras fuentes y dispositivos. Por tanto, se configura como un espacio abierto para que los usuarios vivan experiencias y las compartan. 
Concretamente, el proyecto Teatro en red nace de la mano de dos docentes que pretenden expandir el teatro contemporáneo entre el alumnado. Para ello, han creado un blog con un alto grado de rigurosidad, en el que comparten tanto experiencias didácticas como obras teatrales de actualidad para animar a los jóvenes en el ámbito teatral.

En suma y en acuerdo con Pérez Fernández (2011), Santos Rodríguez y FernándezRío (2012), creemos que teatro y blog son dos realidades que convergen en un mismo punto para la innovación, la mejora y la calidad de la enseñanza y de los procedimientos lectores.

\section{Método}

Atendiendo a Flick (2015), la investigación cualitativa nos ha posibilitado conocer un instrumento educativo y sus características, así como sus finalidades para la promoción lectora. En este sentido, el principal objetivo ha sido examinar y comprender cómo el blog de los docentes permite el fomento y la difusión de los textos teatrales entre el público. Paralelamente, hemos establecido los siguientes objetivos específicos: describir la perspectiva de los profesores sobre el blog y sus servicios para la comunidad educativa; entender cómo el blog es un recurso para la difusión y lectura de obras dramáticas; explicar los beneficios del blog como herramienta lectora; y trazar la arquitectura del blog al servicio del teatro contemporáneo y la lectura.

En este estudio, han participado dos docentes de Lengua castellana y Literatura de la Comunidad Autónoma de Galicia. Ambos poseen una dilatada trayectoria en la enseñanza y en el campo teatral y, además, mantienen un fuerte compromiso por la difusión de la lectura de textos teatrales contemporáneos. Por este motivo, el proyecto Teatro en red tiene como propósito crear una concienciación lectora general y globalizada entre la población.

De acuerdo con González Sanmamed (1995), Simons (2011), y Kvale (2011), entendemos que la entrevista, como técnica de recogida de datos, nos aproxima a una comprensión del objeto de estudio desde la óptica de los participantes del fenómeno social. En este caso, se aplicó a los dos profesores participantes una entrevista de tipo cualitativo y estructurada en tres bloques con un total de 20 preguntas. Por otra parte, en línea con Rapley (2014), hemos atendido al análisis de documentos para examinar el blog de los docentes y, al mismo tiempo, con el fin de discernir aquellos aspectos que promuevan la lectura de textos dramáticos.

Tal y como establecen Miles y Huberman (1994) y Gibbs (2012), hemos realizado un análisis sistemático y codificado. Para ello, hemos perfilado el siguiente esquema de análisis. Véase la figura 1:

ISL, vol. 5, 2016, págs. 44-51 ISNN: 2340-8685
Blanco Martínez, A (2016): Promover la lectura de obras teatrales a través del blog docente Teatro en red, Investigaciones Sobre Lectura, 5, 44-51. 


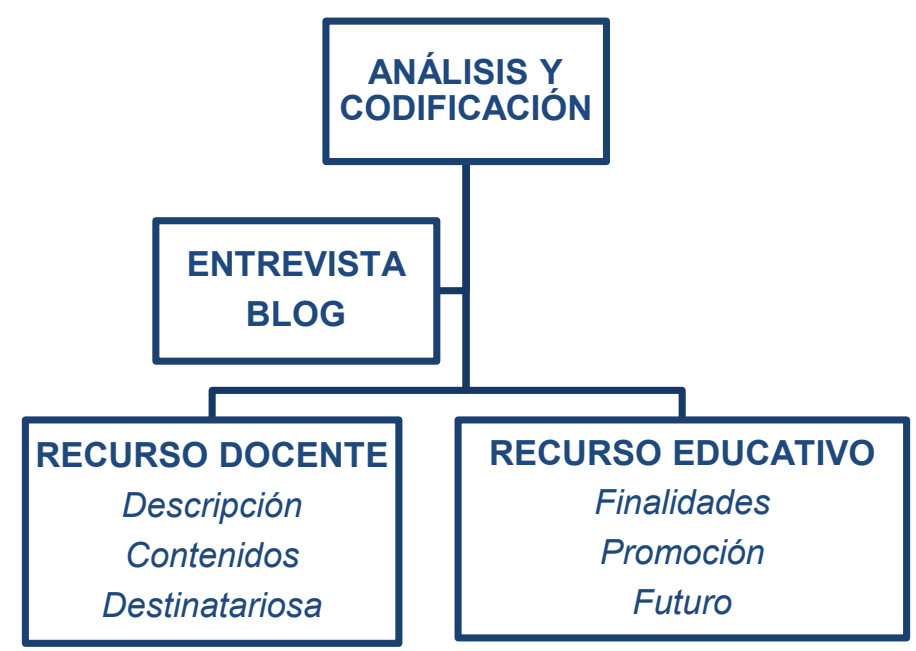

Figura 1. Esquema de codificación y análisis de los datos

\section{Resultados}

A continuación, esbozamos los resultados obtenidos. Para ello, hemos establecido dos dimensiones referidas al proyecto de estudio: el blog como recurso de los docentes y el blog como herramienta para la promoción lectora en contextos educativos.

\subsection{Recurso docente}

El proyecto Teatro en red $^{1}$ posee una arquitectura muy accesible para cualquier usuario con interés en ahondar en la lectura de textos teatrales contemporáneos. Tal y como nos explica la profesora B "el blog presenta textos de interés para los jóvenes y actualizados" (p. 2) y se estructura en "reseñas literarias de obras de teatro contemporáneo, guías de lectura, propuestas didácticas" (p. 4).

En el análisis de la plataforma, hemos identificado una serie de secciones muy válidas para promover entre los jóvenes una lectura online e inmediata, así como una lectura más personal. A continuación, presentamos una tabla en donde recogemos estos contenidos. Véase la tabla 1:

\begin{tabular}{l|l}
\hline SECCIONES & \multicolumn{1}{c}{ CARACTERÍSTICAS } \\
\hline \multirow{4}{*}{$\begin{array}{l}\text { Guías de } \\
\text { lectura }\end{array}$} & $\begin{array}{l}\text { Sinisterra (El lector por horas); Juan Mayorga (Himmelweg, El chico de la última fila, } \\
\text { La tortuga de Darwin); Olga Mínguez (El atardecer de cristal); Fernando J. López } \\
\text { (Cuando fuimos dos); H. Ibsen (Casa de muñecas); Carmen Varela Pérez (El juego de }\end{array}$ \\
& $\begin{array}{l}\text { conocerse); Teresa Calo (El hombre que sobremurió a una mudanza); Ignacio } \\
\text { Aranguren (Pícaros cómicos clásicos). }\end{array}$ \\
\hline
\end{tabular}

${ }^{1}$ Enlace del blog: https://elblogdeteatroenred.wordpress.com/

Blanco Martínez, A (2016): Promover la lectura de obras teatrales a través del blog docente Teatro en red, Investigaciones Sobre Lectura, 5, 44-51.
ISL, vol. 5, 2016, págs. 44-51 ISNN: $2340-8685$ 


\begin{tabular}{l|l}
\hline $\begin{array}{l}\text { Unidades } \\
\text { didácticas }\end{array}$ & $\begin{array}{l}\text { Conjunto de actividades y propuestas para que los jóvenes reflexionen sobre el valor } \\
\text { fines del teatro en la sociedad. Entre ellas, podemos citar: La mirada del hombre } \\
\text { oscuro y Oseznos de Ignacio del Moral, La casa de Bernarda Alba de Federico García } \\
\text { Lorca, Las redes sociales y el teatro, El teatro es para la ESO. }\end{array}$ \\
\hline Crítica teatral & $\begin{array}{l}\text { Noticias teatrales, el radio teatro como formato teatral, vinculación entre teatro y } \\
\text { ciencias, artículos sobre crisis teatral, estrenos actuales, artículos científicos sobre } \\
\text { teatro como recurso educativo, etc. }\end{array}$ \\
\hline
\end{tabular}

En cuanto a los destinatarios del blog, ambos participantes consideran que se trata de una red con libre acceso y dirigida a todo el público interesado en el teatro. La profesora B nos explica que "recomienda su uso a un público mayor de 10 años hasta otros más complejos" (p. 1). Por su parte, el docente A nos comenta que es "una plataforma destinada a cualquier individuo de la educación formal y no formal” (p. 1).

\subsection{Recurso educativo}

Los investigados conciben el blog como instrumento facilitador para promover intereses lectores entre los jóvenes. El profesor A concibe dicho recurso como "una herramienta para el intercambio de información sobre obras dramáticas y para la orientación en su lectura" (p. 1), mientras que la docente B subraya el blog "como vía para la divulgación de contenidos y experiencias" (p. 1).

Ambos profesores consideran que los estudiantes tienen un total desconocimiento sobre el teatro y su vertiente para la lectura y el entretenimiento. La participante B cree que "no hay una cartelera teatral pensada para un público adolescente lo suficientemente atractiva, abundante y de ahí que no se explote su posible interés entre nuestros jóvenes y, por tanto, ellos tampoco la puedan conocer" (p. 3). Por este motivo "emerge el proyecto Teatro en red, es decir, para suplir este vacío en la sociedad” (A, p. 3).

En lo que la promoción lectora se refiere, nos relatan que "hacemos muchas recomendaciones de lecturas, procuramos compartir recursos de webs donde encontrar lecturas teatrales atractivas, sugerimos editoriales que trabajen el teatro para adolescentes con la intención de conseguir nuevos lectores para este género" (B, p. 4). Además, "la red social nos permite mayor repercusión debido a su capacidad de difusión e inmediatez" (A, p. 3). Así, se consigue que los estudiantes "perciban que el blog es una red social perfecta para la lectura, el aprendizaje, el conocimiento y diversión” (B, p. 9).

Con perspectiva de futuro, se pretende ampliar los horizontes del blog para que haya una participación más activa de los jóvenes y puedan plasmar sus impresiones sobre los textos de lectura. En este sentido, la profesora B nos comenta que lo ideal "sería añadir un espacio en el cual participasen directamente los adolescentes subiendo sus propias reseñas sobre lecturas teatrales realizadas" (p. 12). Se trata de un camino "para conseguir

ISL, vol. 5, 2016, págs. 44-51 ISNN: 2340-8685
Blanco Martínez, A (2016): Promover la lectura de obras teatrales a través del blog docente Teatro en red, Investigaciones Sobre Lectura, 5, 44-51. 
un público lector activo y participativo, además de lograr que aumente el placer por leer y compartir textos teatrales" (B, p. 15).

También, cabe señalar que actualmente los investigados están trabajando en la creación de un club virtual de lectura para "una incorporación masiva de los estudiantes en el blog” (A, p. 12). Este planteamiento permitiría más posibilidades didácticas y lectoras, bajo "la supervisión de un profesor intermediario" (A, p. 13).

En último término, el profesor A comenta que todavía no se ha conseguido un mayor impacto en los estudiantes "porque el docente no busca información sobre teatro y segundo, porque se muestra reacio a alejarse de la programación oficial y de los libros de lectura obligatorios" (p. 16). Por este motivo, la participante B sugiere que "hay concienciar de que la lectura de textos dramáticos sirve y mucho para provocar oralidad y reflexividad en los estudiantes, así como un espíritu crítico" (p. 19).

\section{Discusión}

Asumiendo los aportes teóricos de Flick (2015), la utilización de la investigación cualitativa nos ha aportado una visión específica sobre la acción y visión de dos docentes comprometidos con el teatro $\mathrm{y}$, de la misma manera, sobre el funcionamiento y las repercusiones del blog en la comunidad educativa y en la sociedad lectora. De acuerdo con los objetivos previamente formulados, planteamos las siguientes conclusiones.

En primer lugar, cabe decir que la perspectiva de los profesores nos ha clarificado los propósitos y finalidades del proyecto de estudio. Hemos comprendido que el blog tiene como prioridad hacer llegar el teatro a los jóvenes y, al mismo tiempo, publicitar y expandir las novedades teatrales que están a su alcance.

Por otra parte, el análisis de la red social y de su formato arquitectónico nos ha revelado los propósitos lectores que persigue. En este sentido, dado el valor que tienen los recursos TIC en la sociedad actual, se convierten en espacios con una rápida repercusión para producir cambios en los individuos y, así, poder orientarlos hacia el gusto teatral.

En lo que respecta a los beneficios del blog como herramienta de lectura, cabe resaltar el fácil acceso desde cualquier parte y a cualquier hora, siempre y cuando se tenga conexión con alguna red. Además, posibilita que los estudiantes accedan a información rigurosa y confíen en los criterios y sugerencias lectoras de profesionales de la educación.

En último lugar, en lo que se refiere a la pura descripción del blog, nos ha valido para aprender sobre cómo organizar y distribuir contenidos teatrales en la red. Se trata 
crear un espacio de fácil acceso e intuitivo, para que los usuarios concentren las energías en la lectura y en el aprendizaje teatral.

En conclusión y en sintonía con Hernández Sellés (2015), es importante crear espacios virtuales para el aprendizaje y para la colaboración entre los internautas. En suma, hay que atender a las demandas sociales para proporcionar lugares seguros para la lectura, el deleite y el enriquecimiento personal.

\section{Bibliografía:}

Cabero, J. (2010). Los retos de la integración de las TIC en los procesos educativos: límites y posibilidades. Perspectiva Educacional, 49 (1), 32-61.

Díez Mediavilla, A. y García Velasco, A. (2015). La competencia lectora: perfiles y artistas. Revista Lenguaje y Textos, 41, 5-8.

Flick, U. (2015). El diseño de la investigación cualitativa. Madrid: Morata.

Gibbs, G. (2012). El análisis de datos cualitativos en investigación cualitativa. Madrid: Morata.

González Sanmamed, M. (1994). Aprender a enseñar: mitos y realidades. A Coruña: Servicio de Publicaciones de la Universidad de A Coruña.

Hernández Serrano, Ma . J. y González Sánchez, M. (2014). Las posibilidades del blog como elemento de apoyo y refuerzo en el aprendizaje. En A. García-Valcárcel y Muñoz-Repiso (Coord.), Experiencias de innovación docente universitaria. (pp. 369-374). Salamanca: Aquilafuente.

Hernández Sellés, N. (2015). El trabajo colaborativo en entornos virtuales en educación superior. (Tesis Doctoral Inédita). Universidad de A Coruña.

Jiménez Pérez, E. (2015). Niveles de comprensión y la competencia lectoras. Revista Lenguaje y Textos, 41, 19-26.

Kvale, S. (2011). Las entrevistas en investigación cualitativa. Madrid: Morata.

Miles, M.B. y Huberman, A.M. (1994). Qualitative Data Analysis: An expanded sourcebook. Thousand Oaks, California: SAGE.

Pérez Fernández, J. (2011). Motivar en secundaria. El teatro: una herramienta eficaz. Barcelona: Ediciones Erasmus.

Rapley, T. (2014). Los análisis de conversación, de discurso y de documentos en investigación cualitativa. Madrid: Morata.

ISL, vol. 5, 2016, págs. 44-51 ISNN: 2340-8685
Blanco Martínez, A (2016): Promover la lectura de obras teatrales a través del blog docente Teatro en red, Investigaciones Sobre Lectura, 5, 44-51. 
Simons, H. (2011). El estudio de caso. Teoría y práctica. Madrid: Morata.

Santos Rodríguez, L. y Fernández-Río, J. (2012). Uso pedagógico del blog: un proyecto de investigación-acción en la materia de Educación Física en Educación Secundaria. EDUTEC. Revista Electrónica de Tecnología Educativa, 42. Recuperado el 12 de enero, 2016 de http://edutec.rediris.es/. 